\title{
OPEN Metabolomic analysis of Trichophyton rubrum and Microsporum canis during keratin degradation
}

\author{
Anita Ciesielska ${ }^{1}{ }^{凶}$, Anna Kawa ${ }^{1}$, Katarzyna Kanarek ${ }^{1}$, Adrian Soboń ${ }^{1}{ }^{1}$ \& \\ Rafał Szewczyk (D) $^{2}$
}

Keratin is important and needed for the growth of dermatophytes in the host tissue. In turn, the ability to invade keratinised tissues is defined as a pivotal virulence attribute of this group of medically important fungi. The host-dermatophyte interaction is accompanied by an adaptation of fungal metabolism that allows them to adhere to the host tissue as well as utilize the available nutrients necessary for their survival and growth. Dermatophyte infections pose a significant epidemiological and clinical problem. Trichophyton rubrum is the most common anthropophilic dermatophyte worldwide and its typical infection areas include skin of hands or feet and nail plate. In turn, Microsporum canis is a zoophilic pathogen, and mostly well known for ringworm in pets, it is also known to infect humans. The aim of the study was to compare the intracellular metabolite content in the $T$. rubrum and $M$. canis during keratin degradation using liquid chromatography system coupled with tandem mass spectrometer (LC-MS/MS). The metabolite "fingerprints" revealed compounds associated with amino acids metabolism, carbohydrate metabolism related to the glycolysis and the tricarboxylic acid cycle (TCA), as well as nucleotide and energy metabolism. The metabolites such as kynurenic acid, L-alanine and cysteine in case of $T$. rubrum as well as cysteine and riboflavin in case of $M$. canis were detected only during keratin degradation what may suggest that these compounds may play a key role in the interactions of $T$. rubrum and $M$. canis with the host tissue. The metabolomic results were completed by qPCR gene expression assay. Our findings suggest that metabolomic analysis of $T$. rubrum and $M$. canis growing in culture media that mimic the dermatophyte infection could allow the understanding of processes involved in the pathogenesis of dermatophytes.

Dermatophytes such as Trichophyton rubrum and Microsporum canis belong to a group of filamentous fungi infecting keratinised structures, including the skin, hair, and nails. T. rubrum is representative of anthropophilic fungi and responsible for over $60 \%$ of all dermatophytosis such as Tinea pedis, Tinea corporis, Tinea inguinalis, Tinea unguium as well as deep dermal infections ${ }^{1}$. M. canis is one of the main etiological agents of dermatophytosis in animal mainly cats and dogs. However, this zoophilic dermatophyte can be transmitted to humans through direct contact with infected animal via arthrospores ${ }^{1}$. A keratinolytic activity and pathogenesis of this group of medically important fungi is corelated because, during infection, dermatophytes secrete a lot of proteases that degrade keratinised structures into oligopeptides as well as free amino acids, which are then used by the fungi as sources of nutrients ${ }^{2}$. Proteolytic degradation of keratin is possible after relaxation of its structure by reduction of disulphide bridges (S-S). Sulphite $\left[\mathrm{SO}_{3}{ }^{2-}\right]$ is excreted by the sulphite efflux pump SSU1, and acts as a reducing agent. The loosened structure of the keratin makes peptide bonds more accessible for digestion by the secreted proteases. Cooperation between the proteases and reducing agent results in the formation of smaller peptides and amino acids that can be taken up by the fungal cell. Cysteine, created during the decomposition of keratin, is toxic, but can be metabolised to sulphite by the action of the enzyme cysteine dioxygenase type 1 (encoded by CDO1), and the sulphite formed is excreted by the fungal cell again, which further facilitates keratin degradation $^{3,4}$. Thus, the ability to degrade keratin is one of the important virulence attributes of these pathogens.

Metabolomics, following transcriptomics and proteomics, plays an important role in the understanding of the mechanisms of bacterial $^{5-7}$, fungal ${ }^{8,9}$ or viral ${ }^{10,11}$ pathogenesis and becoming very useful in life sciences. 


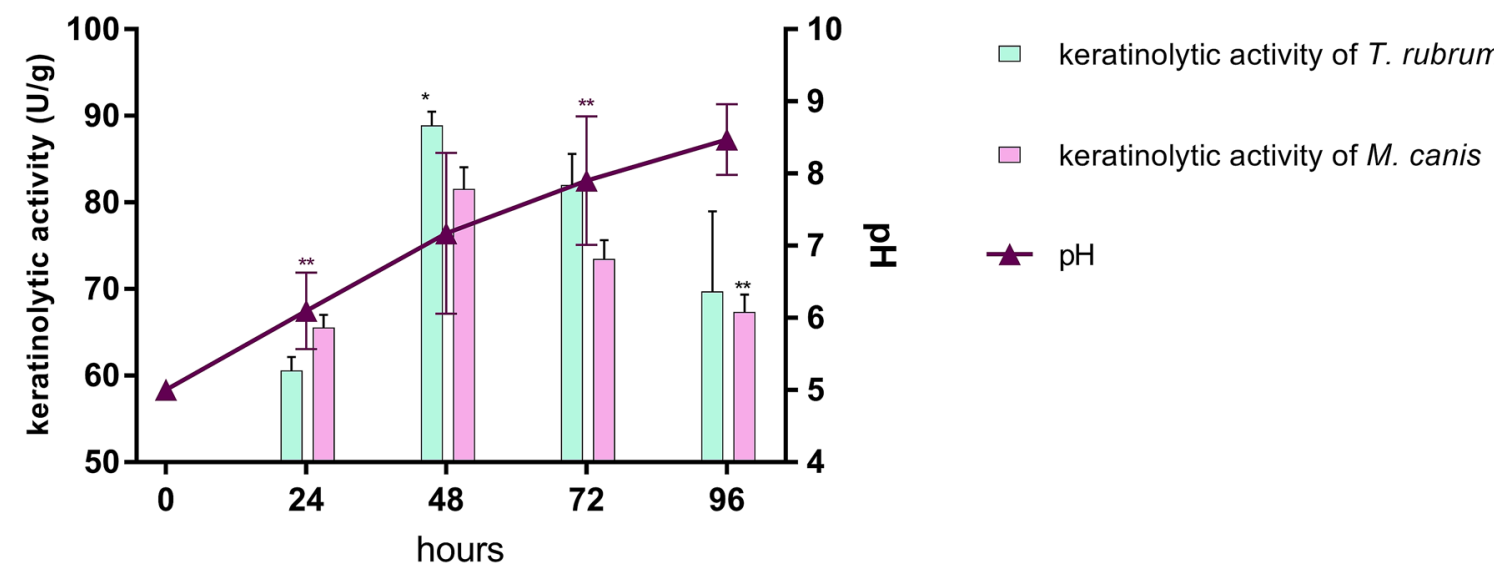

Figure 1. Evaluation of keratinolytic activity and $\mathrm{pH}$ changes of T. rubrum and $M$. canis at $30^{\circ} \mathrm{C}$ in the presence of keratin. Keratinolyctic activity was calculated in unities/g $(\mathrm{U} / \mathrm{g})$. The bars in the columns and triangles represents the standard deviation of data obtained from three independent replicates. Significant statistical differences are represented by asterisks $\left({ }^{*} p<0.5 ;{ }^{* *} p<0.01\right)$.

Compared to the genome and transcriptome, the proteome and metabolome are much more complex and dynamic $^{12,13}$. The gene expression does not always reflect directly in the amount and activity of compounds such as proteins and metabolites in the cells ${ }^{14}$. However, by comparing profiles of metabolites or proteins of control samples with samples growing under specific conditions, information about qualitative and quantitative changes, induced by the stimuli factor, is obtained ${ }^{15}$. Mass spectrometry (MS) is the basic omics method of choice in case of metabolomic studies ${ }^{8}$. This analytical tool is very useful for a better understanding of the biology of organisms and their response to different environmental stimuli ${ }^{16}$. Cellular processes are characterised by the production of unique, chemical "fingerprints", a set of metabolites with specific compositions ${ }^{17}$. Thus, in the present study, liquid chromatography with tandem mass spectrometry (LC-MS/MS) based, untargeted metabolomic analysis of T. rubrum and M. canis cells, was performed. The cells grew in the presence of a two carbon sources such as keratin, which mimic the dermatophyte infection to host cells and glucose. Determination of characteristic metabolites of these two dermatophytes originated from different niches may expand knowledge about their pathogenic properties.

\section{Results}

Keratinolytic activity of T. rubrum and $M$. canis and the $\mathrm{pH}$ changes. The obtained results revealed that T. rubrum and M. canis isolates have ability to degraded keratin. The production of keratinolytic enzyme was found at its maximum of $88.9 \mathrm{U} / \mathrm{g}$ in case of the culture filtrate of T. rubrum and $81.6 \mathrm{U} / \mathrm{g}$ in case of the culture filtrate of $M$. canis (Fig. 1) after $48 \mathrm{~h}$ of incubation. The initial $\mathrm{pH}$ of the keratin medium during growth of $T$. rubrum and M. canis increased from 5.0 to 8.7 after $24-96 \mathrm{~h}$ of incubation (Fig. 1). The differences between these averages was statistically significant $(p<0.05)$.

Metabolomic analysis of T. rubrum and M. canis. Quantitative analysis of intracellular metabolites of T. rubrum and M. canis obtained by separation using the LC-MS/MS method, was performed using PCA and conditional formatting in Microsoft Excel. A total of 90 intracellular metabolites (Table S1) from T. rubrum and $M$. canis were measured after $48 \mathrm{~h}$ incubation in minimal medium (MM-Cove) supplemented with glucose or keratin as a carbon sources, across the three biological and technical replicates. A 62 and 58 compounds were identified in T. rubrum and M. canis respectively, and noticeable interspecific differences between the metabolites, were observed. The PCA detected metabolites presenting statistically significant differences, between the two experimental conditions (Fig. 2A,C). Figure 2B,D presents the distribution of individual metabolites in 2D PCA scores plots. In case of T. rubrum, detected metabolites, such as L-proline, betaine, and malic acid with spectra on the left side of the graph, presented higher concentrations in the control medium, while intracellular metabolites, such as L-glutamine, L-tryptophan, and L-tyrosine, with spectra located on the right side of the graph, presented higher concentrations in the medium supplemented with keratin (Fig. 2D). In case of M. canis, detected metabolites such as asparagine, L-tyrosine, L-threonine or L-aspartic acid with spectra on the left side of the graph presented higher concentrations in the medium supplemented with keratin. However, metabolites such as L-alanine, L-proline, and reduced and oxidised glutathione with spectra on the right side of the graph, presented higher concentrations in the control medium (Fig. 2B). Other metabolites such as histidine, L-leucine, and L-phenylalanine in case of T. rubrum as well as isocitric acid and cytidine-5'-monophosphate in case of $M$. canis, whose spectra were in the centre of the graph, showed similar concentration ratios for the control medium, and the medium supplemented with keratin (Fig. 2B,C).

In case of T. rubrum, among 62 intracellular metabolites (Fig. 3), 45 (73\%) were marked as being produced more intensively in the control medium, seven of them, such as hydroxy-L-proline, nicotinic acid, oxaloacetate, uracil, $\gamma$-aminobutyric acid (GABA), $p$-aminobenzoic acid (PABA), and guanosine- 5 '-diphosphate (GDP) were detected only in the control medium. In turn, 17 compounds (27\%) were detected as being produced more intensively in the medium supplemented with keratin, and three out of these, L-alanine, kynurenic acid and 
Microsporum canis
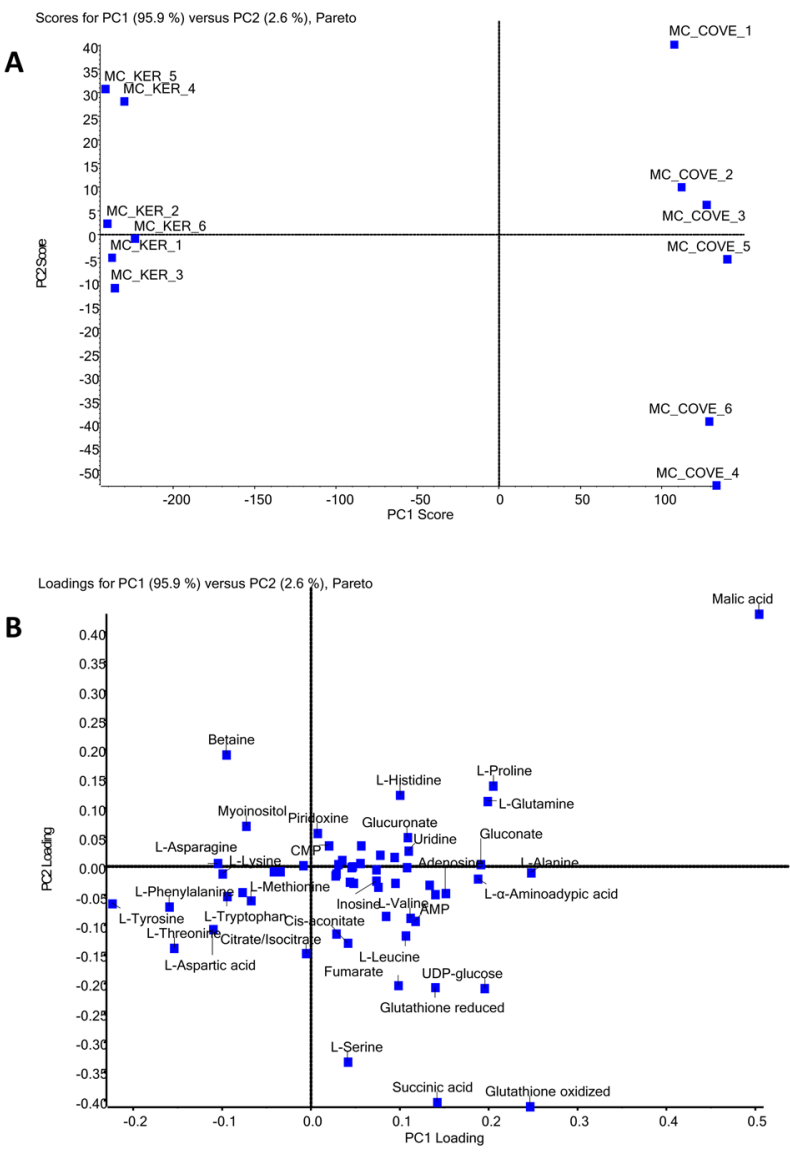

Trichophyton rubrum
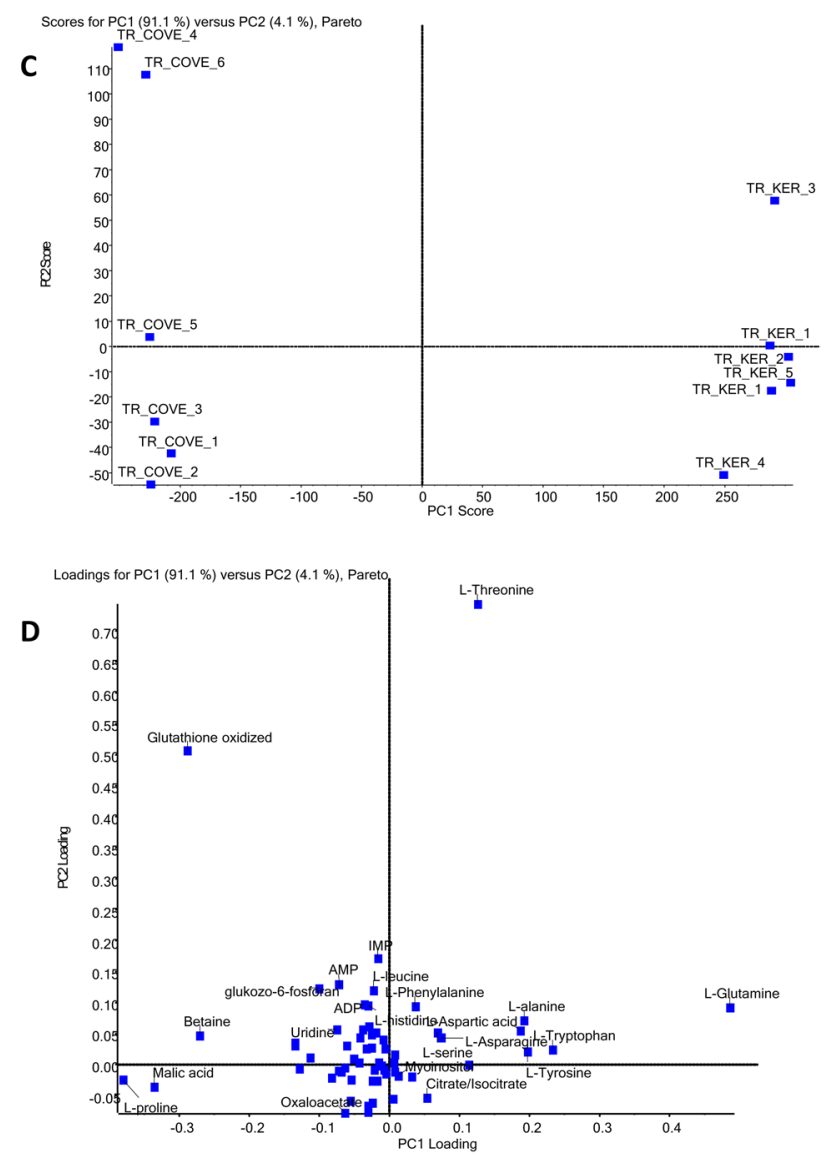

Figure 2. $(\mathbf{A}, \mathbf{C}) \mathrm{PCA}$ of the intracellular metabolites in minimal medium supplemented with glucose (TR-COVE, MC-COVE) or keratin (TR-KER, MC-KER) as a carbon sources (B,D) 2D PCA scores plots indicate statistically significant sample separation along the first dimension (PC1).

cysteine were present only in this growth environment. Two of the three vitamins found, namely nicotinic acid and PABA, were classified as specific to the control medium, while the third, riboflavin is produced more intensively in the presence of keratin. Increased synthesis of amino acids such as L-alanine, L-tyrosine, aspartic acid, L-tryptophan, and glutamine was observed when the fungus degraded keratin present in the medium (Fig. 3).

Among the 58 identified intracellular metabolites in M. canis (Fig. 3), 42 compounds (72\%) were up-regulated in the control medium, while 16 metabolites presented higher concentration values in the medium supplemented with keratin. Six compounds namely L-alanine, diaminopimelic acid (DAP), reduced glutathione, oxidised glutathione, guanosine-5'-triphosphate (GTP), and pyridoxine (vitamin B6) were detected only in the control medium, while two compounds (Fig. 3), cysteine and riboflavin were detected only in the medium supplemented with keratin. A reduced concentration of amino acids, TCA-and glycolysis-related metabolites, and nucleotides was observed in the medium supplemented with keratin. The fungus intensively produced, intracellular metabolites such as GABA, L-tyrosine, L-phenylalanine, L-methionine, and phosphoenolpyruvic acid (Fig. 3) in the medium supplemented with keratin.

Validation of metabolomic results by quantitative polymerase chain reaction (qPCR) analysis. The validation was performed using templates from the T. rubrum CBS 120358 and M. canis CBS 11348 strains, incubated at $28^{\circ} \mathrm{C}$ for $48 \mathrm{~h}$ in the control medium and in the medium supplemented with keratin. Four genes for T. rubrum and four genes for M. canis (Table 1) encoding the identified metabolites (Fig. 3) were randomly selected. Kynurenine aminotransferase (TERG_07193) catalyses the synthesis of kynurenic acid in the kynurenine pathway, while citrate synthase (TERG_04125, MCYG_06837) is a key enzyme of the TCA cycle, catalysing the synthesis of citric acid from acetyl-CoA. Enolase (TERG_01612, MCYG_07208) known as phosphopyruvate hydratase is a glycolytic enzyme responsible for the catalysis of conversion of 2-phosphoglycerate to phosphoenolpyruvate (PEP). Riboflavin synthase (TERG_00247, MCYG_00702) is an enzyme that catalyses the final reaction of riboflavin synthesis. SSU1 gene (MCYG_08415) was found to be strongly activated during the growth of dermatophyte cells in the presence of cysteine. The qPCR data (Fig. 4) and the corresponding metabolomic results (Figs. 3, 5) from the three biological replicates confirmed the reliability of the metabolomic data obtained (Pearson's correlation, $r>0.97, p<0.001$ ). 

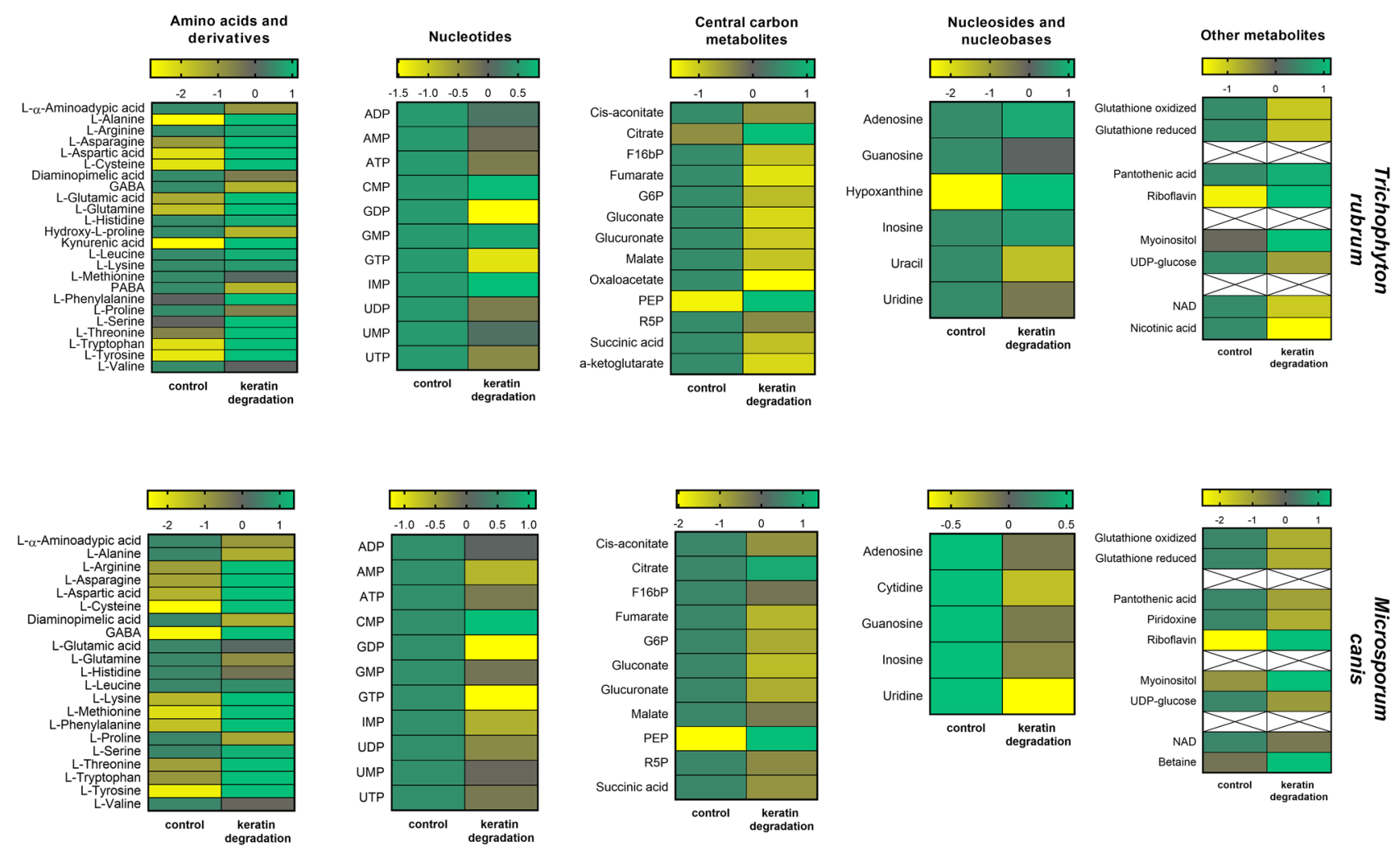

Figure 3. Heat map visualisation for all detected intracellular metabolites of T. rubrum and M. canis in control medium and during keratin degradation.

\section{Discussion}

Trichophyton rubrum and Microsporum canis are group of fungi classified as anthropophilic and zoophilic, respectively, what is related to their natural habitat ${ }^{1}$. These two dermatophyte species differ also in host range as well as dermatophytosis progression. Moreover, both of them during infection can evade the host immune system what leads to the chronic infections ${ }^{18}$. Previously study about a comparative genome analysis of five dermatophytes species such as T. rubrum, T. tonsurans, T. equinum, M. canis and M. gypseum revealed some interspecific differences between them, what suggested that various gene as well as post-transcriptional regulation are known as the dermatophyte arsenal necessary to survive in the various niches and different hosts ${ }^{19}$. Extending of studies to metabolomic analysis to compare other interspecies differences are required to complete answers to the questions how these fungal pathogens adapt to different niches as well as how they utilize the available nutrients necessary for its survival and growth during the host-dermatophyte interaction.

The beginning of any dermatophyte infection in vivo is in the form of a direct contact of the pathogen cells with the target host structure. If the current condition of the host is conducive to the initiation of infection, arthroconidia of dermatophytes adhere to keratinised tissues and begin their penetration process ${ }^{20}$. Dermatophytes produce a broad spectrum of proteolytic enzymes which are essential to their pathogenicity as well as virulence, are the key proteins involved in keratin degradation ${ }^{21}$. Degradation of keratin by dermatophytes releases amino acids, whose metabolism leads to secretion of ammonia, which changes the initial $\mathrm{pH}$ in the host tissue from acidic ( $\mathrm{pH}$ 5.0) to alkaline ( $\mathrm{pH} 8.5$ ) approximately within 72-96 h, producing an environment in which most of the known keratinolytic proteases of dermatophytes have optimal enzymatic activity. This in turn is extremely important for the growth of the dermatophytes in the host tissue $e^{2,21-26}$. In this work, we reported that T. rubrum and M. canis have the highest keratinolytic activity at $48 \mathrm{~h}$ time point. Our results demonstrated also that the optimum $\mathrm{pH}$ for keratinases production was slightly alkaline ( $\mathrm{pH}$ 7.2) (Fig. 1) what agreed with the previously reported data ${ }^{2,21-24}$. Therefore, we decided to choose the measuring point at $48 \mathrm{~h}$, to identify intracellular metabolites in T. rubrum and M. canis, during the degradation of keratin by these fungi. The keratin obtained from sheep wool, human hair or bird feathers is characterised by a high content of amino acids such as (1) non-ionic polar amino acids, for e.g., serine, cysteine, threonine, and tyrosine; (2) ionic polar amino acids, for e.g., arginine, lysine, aspartic acid, and glutamic acid; and (3) non-polar amino acids, for e.g., alanine, glycine, leucine, methionine, phenylalanine, proline, and valine $\mathrm{e}^{27}$. All these amino acids were detected, in the present study, in T. rubrum as well as in M. canis, in the control medium, and in the medium supplemented with keratin (Figs. 3, 5). After $48 \mathrm{~h}$, a significant increase in the amount of intracellular amino acids and their derivatives were observed, when T. rubrum and M. canis degraded keratin (Figs. 3, 5) which can then be used as a sources of carbon, nitrogen as well as sulphur ${ }^{2,28,29}$.

Compounds such as L-alanine, kynurenic acid and cysteine in case of T. rubrum, as well as cysteine and riboflavin in case of $M$. canis were detected only in the medium supplemented with keratin (Figs. 3, 5). The significant increase in the production of kynurenic acid by T. rubrum in the medium which promotes keratin degradation 


\begin{tabular}{|c|c|c|c|c|c|c|}
\hline Accession no. & Gene product name & $\begin{array}{l}\text { Primers }\left(5^{\prime}-3^{\prime}\right) \\
\text { Forward } \\
\text { Reverse }\end{array}$ & $\begin{array}{l}\text { Length } \\
{[\text { bp] }}\end{array}$ & $\operatorname{Tm}\left[{ }^{\circ} \mathbf{C}\right]$ & Efficiency (\%) & $\mathbf{R}^{2}$ \\
\hline \multirow{2}{*}{ TERG_07193 } & \multirow{2}{*}{ Kynurenine aminotransferase } & $\begin{array}{l}\text { GCAAGCAACGAGCCTTTC } \\
\text { AA }\end{array}$ & \multirow{2}{*}{196} & \multirow{2}{*}{60.5} & \multirow{2}{*}{101} & \multirow{2}{*}{0.99814} \\
\hline & & $\begin{array}{l}\text { GGTTAGACTCCACGGTCT } \\
\text { GC }\end{array}$ & & & & \\
\hline \multirow{2}{*}{ TERG_04125 } & \multirow{2}{*}{ Citrate synthase } & $\begin{array}{l}\text { GGCTCCTACCCTCAAGGA } \\
\text { GA }\end{array}$ & \multirow{2}{*}{195} & \multirow{2}{*}{60.5} & \multirow{2}{*}{100} & \multirow{2}{*}{0.99755} \\
\hline & & $\begin{array}{l}\text { CGGAAGCGAATACCCTCC } \\
\text { TC }\end{array}$ & & & & \\
\hline \multirow{2}{*}{ TERG_01612 } & \multirow{2}{*}{ Enolase } & \begin{tabular}{|l} 
GAAGGGTGTCCCACTGTA \\
CG
\end{tabular} & \multirow{2}{*}{132} & \multirow{2}{*}{60.5} & \multirow{2}{*}{97} & \multirow{2}{*}{0.99675} \\
\hline & & $\begin{array}{l}\text { AACTCCTGGAAAGCGAGA } \\
\text { CG }\end{array}$ & & & & \\
\hline \multirow{2}{*}{ TERG_00247 } & \multirow{2}{*}{ Riboflavin synthase } & $\begin{array}{l}\text { GGGCGGGACATCCTTAAC } \\
\text { AA }\end{array}$ & \multirow{2}{*}{190} & \multirow{2}{*}{60.5} & \multirow{2}{*}{102} & \multirow{2}{*}{0.99855} \\
\hline & & $\begin{array}{l}\text { GACTGGTGATCCGGCTTT } \\
\text { GA }\end{array}$ & & & & \\
\hline \multirow{2}{*}{ MCYG_08415 } & \multirow{2}{*}{ SSU1 gene } & \begin{tabular}{|l} 
AAGAGCTTCAGGTCACAG \\
CC
\end{tabular} & \multirow{2}{*}{191} & \multirow{2}{*}{60.5} & \multirow{2}{*}{99} & \multirow{2}{*}{0.99655} \\
\hline & & $\begin{array}{l}\text { AAGCCCGGGAAACTG } \\
\text { GTATG }\end{array}$ & & & & \\
\hline \multirow{2}{*}{ MCYG_06837 } & \multirow{2}{*}{ Citrate synthase } & $\begin{array}{l}\text { GACACAAGGTCCTCGGTG } \\
\text { AG }\end{array}$ & \multirow{2}{*}{146} & & & \\
\hline & & $\begin{array}{l}\text { GAGTTTCTGGCACTCGGG } \\
\text { AA }\end{array}$ & & 60.5 & 108 & 0.99723 \\
\hline МСУ & $\Gamma_{n}$ & $\begin{array}{l}\text { CGGTGTCAGTTTGGCCAT } \\
\text { TG }\end{array}$ & 17 & $\operatorname{Lor}_{-}$ & 100 & Doos \\
\hline MCYG_07208 & Enolase & $\begin{array}{l}\text { AACTCCTGGAAGGCAAGA } \\
\text { CG }\end{array}$ & 171 & 60.5 & 101 & 0.99651 \\
\hline & & $\begin{array}{l}\text { AGCTCCTTTCAGTCACAC } \\
\text { CG }\end{array}$ & 113 & 605 & & \\
\hline MCYG_00702 & Riboflavin synthase & $\begin{array}{l}\text { AGTGCCGTCAAGAGCAAT } \\
\text { GA }\end{array}$ & 113 & 60.5 & 98 & 0.99490 \\
\hline XM_002848521 & ADP ribosylation factor (adp-rf) & $\begin{array}{l}\text { GAATTCTCATGGTCGGTC } \\
\text { TC }\end{array}$ & 104 & 60.5 & 100 & 0.99855 \\
\hline & & AACGTTGAATCCGATGGTG & & & & \\
\hline Pr & Multiubiquitin chain binding & $\begin{array}{l}\text { AGTCCTAGTTACCTTGAC } \\
\text { CG }\end{array}$ & M & $-60-\pi)$ & ${ }^{2}$ & Donon \\
\hline XM_002843632 & protein $1(m b p 1)$ & $\begin{array}{l}\text { CGGTGTTTAAGTGCTAGA } \\
\text { TAGG }\end{array}$ & 123 & 60.5 & 99 & 0.99924 \\
\hline & & $\begin{array}{l}\text { GAGAAGTTCGAGAAGGAA } \\
\text { GC }\end{array}$ & & & & \\
\hline TERG_00548 & Elongation factor 1-alpha $(e f 1-\alpha)$ & $\begin{array}{l}\text { TGACGGTGACATTGTACT } \\
\text { TG }\end{array}$ & 128 & 60.5 & 107 & 0.99803 \\
\hline & & $\begin{array}{l}\text { GGATCTATATTCACGGCT } \\
\text { CG }\end{array}$ & & & & \\
\hline TERG_04033 & Ribosomal protein L2 ( $r p l 2)$ & $\begin{array}{l}\text { TGGATGATGTTCTTCACG } \\
\text { AC }\end{array}$ & 113 & 60.5 & 102 & 0.99484 \\
\hline
\end{tabular}

Table 1. List of target genes and primer sequences for qPCR.

is interesting (Figs. 3, 4, 5). Kynurenic acid depicts strong immunosuppressive properties on vertebrate immune cells, leading to the silencing of their inflammatory action by limiting the production of cytokines, including interleukins (IL) such as IL-4, IL-23, and tumour necrosis factor- $\alpha$ (TNF- $\alpha$ ). Furthermore, it inhibits Th17 lymphocyte differentiation ${ }^{30}$. IL-17 produced by the Th17 cells, plays an important role in activating antifungal response $^{31,32}$. These reports seem particularly interesting, as T. rubrum, as an anthropophilic dermatophyte, has developed a number of strategies to avoid the host immune response, which directly affects the duration of infections caused and the difficulties in their treatment ${ }^{33}$. In turn, L-alanine is one of the key amino acids that constitutes proteins, and its increased intracellular presence found only when T. rubrum degraded keratin (Figs. 3, 5) may suggest that L-alanine is an important component of proteins responsible for the pathogenesis process of the dermatophyte fungi. In case of the highly virulent mutant of another fungal pathogen, Candida albicans pir32 null strain, L-alanine is part of the sequence of the most identified cell wall proteins (CWPs). Analyses conducted on other pathogenic fungi have shown that CWPs play an essential role in the biosynthesis and rearrangements of the cell wall, and are important virulence factors ${ }^{34,35}$. During the metabolomic analyses of T. rubrum and M. canis, cysteine was detected only during the degradation of keratin by these fungi (Figs. 3, 4, 5). Cysteine, a product of keratin degradation, is oxidised by cysteine dioxygenase (CDO) to cysteine sulphuric acid. This compound is a precursor of taurine, or pyruvate and sulphate, which can be reused in the degradation 


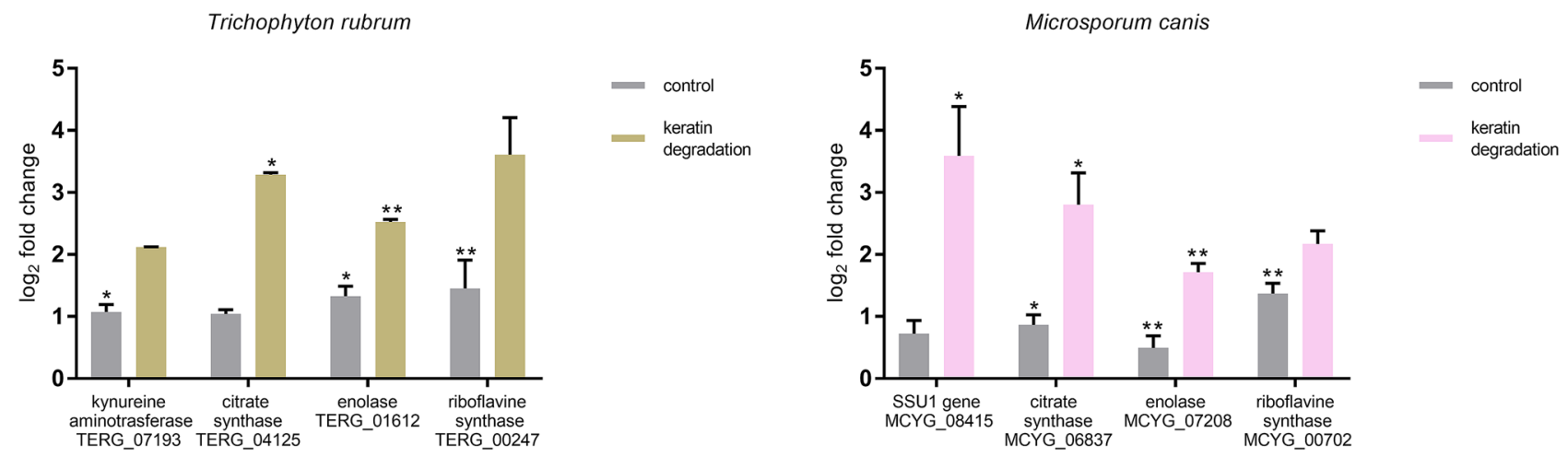

Figure 4. Relative gene expression analysis of selected genes involved in biosynthesis pathways of corresponding metabolites in T. rubrum and M. canis. Asterisks indicate the statistical significance as determined by Student's t-tests $\left({ }^{*} p<0.05 ;{ }^{* *} p<0.001\right)$.

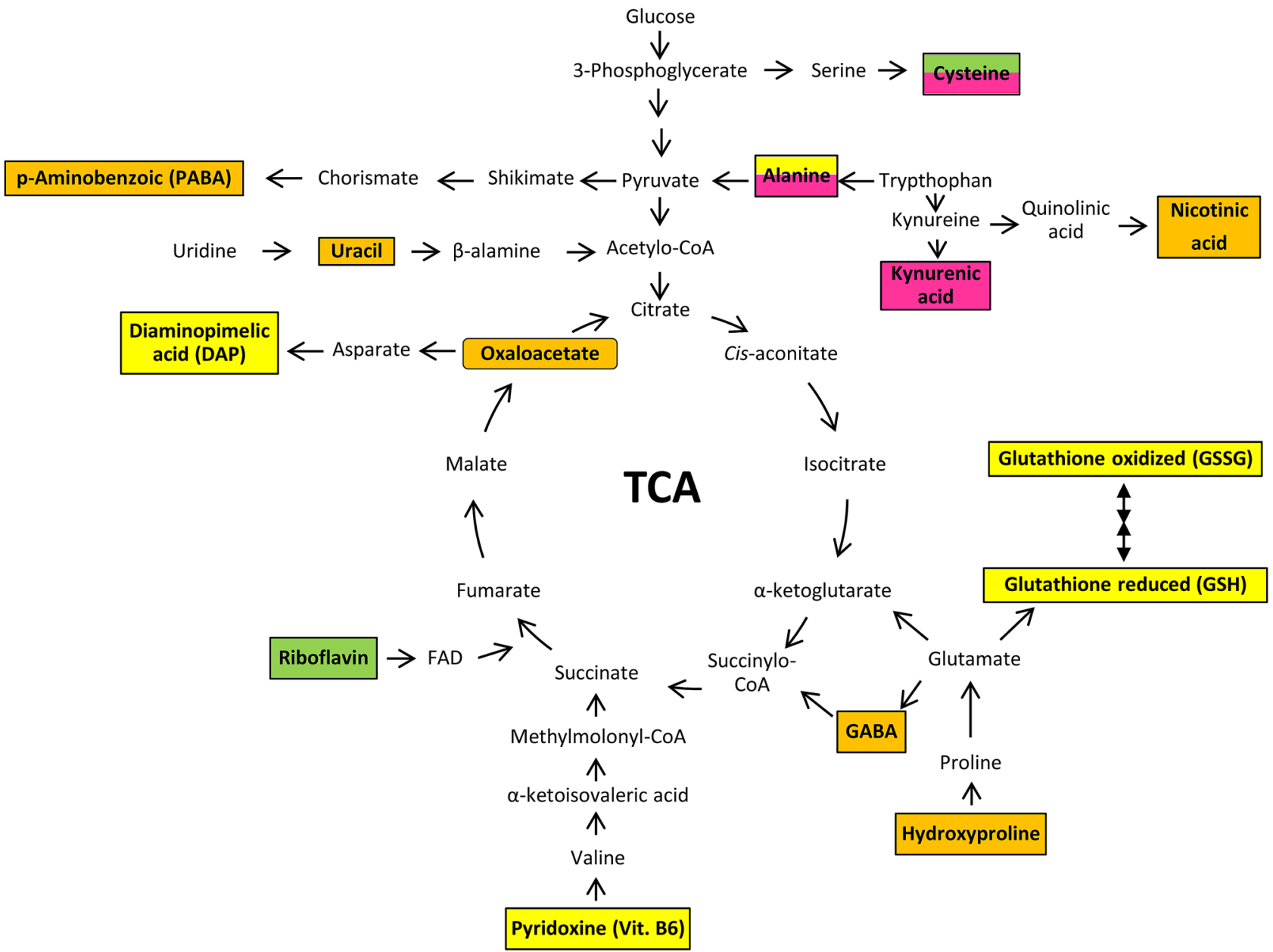

Figure 5. Schematic overview of the metabolomic pathway associated with the detected biochemical compounds. Metabolites of T. rubrum on an orange background represents those marked only in minimal medium (MM-Cove) while metabolites on a pink background represents those marked only during keratin degradation. Metabolites of $M$. canis on a yellow background represents those marked only in minimal medium (MM-Cove) while metabolites on a green background represents those marked only during keratin degradation.

of keratin ${ }^{4}$. Cysteine is a very good source of sulphur and is quickly used up from the medium by the pathogen, even in the presence of better nutrients. Moreover, the excess sulphur is excreted outside the cell in the form of inorganic compounds ${ }^{36}$. The studies on Trichophyton mentagrophytes mutants, which were unable to produce cysteine dioxygenase, showed that the fungus grew very poorly on hair and nails, and was highly sensitive to cysteine $^{3}$. Intracellular cysteine, detected in the present study in T. rubrum and M. canis only in the medium 
supplemented with keratin, confirmed that the pathogens degraded keratin present in the medium and then used it as a substrate for sulphite formation.

Riboflavin (vitamin $\mathrm{B}_{2}$ ) was the second intracellular metabolite that was observed in $M$. canis only during keratin degradation (Figs. 3, 4, 5). Dietl et al. ${ }^{37}$ showed that vitamin $\mathrm{B}_{2}$ is crucial for the pathogenesis of another filamentous fungi Aspergillus fumigatus. The study had revealed that removal of the gene involved in the process of riboflavin biosynthesis $(r i b o B)$ directly results in loss of virulence of this pathogen ${ }^{37}$. Riboflavin is a precursor of flavin mononucleotide (FMN) as well as flavin adenine dinucleotide (FAD), which are cofactors required for catabolic oxidative processes. Flavoproteins produced by A. fumigatus are responsible for the biosynthesis of siderophores or iron assimilation ${ }^{38}$. The virulence of pathogens is often characterised based on the ability of these microorganisms to obtain iron from the environment ${ }^{39}$. Therefore, increased riboflavin production (Figs. 3, 4, 5) by $M$. canis during degradation of keratin may suggest that it is one of the adaptations to the infection process. The biochemical pathway for the synthesis of this metabolite could be a proposed target for an antifungal drug.

It was observed that the growth of T. rubrum and M. canis in the control medium (minimal medium containing glucose as the only carbon source), intensively produced the main products of the intracellular respiration process (Figs. 3, 5). Maranhào et al. ${ }^{40}$ reported that T. rubrum growing in the presence of glucose as the only available carbon source acidifies the medium as well as inhibit the production of external proteases. Glycolysis metabolites can be included in further stages of the process, but they can be alternatively used in other metabolic pathways. For example, the inclusion of glucose-6-phosphate in the pentose phosphate pathway results in the presence of ribose-5-phosphate and nicotinamide adenine dinucleotide phosphate (NADPH) which provides more effective cell protection in conditions of oxidative stress ${ }^{41}$. The minimal medium (MM-Cove) containing glucose as a carbon source used in this study ${ }^{42}$ due to the limited nutrient availability for T. rubrum as well as $M$. canis may lead to increased production of specific protective substances and mechanisms by the microorganism ${ }^{43}$. Moreover, the increased number of metabolites that act as energy transporters, such as GTP, adenosine triphosphate (ATP) or uridine-5'-triphosphate (UTP) in the control medium (Fig. 3) can be useful for the increased synthesis of molecules which have a protective effect on T. rubrum and M. canis cells. In this context, it is worth focusing on hydroxy-L-proline produced by T. rubrum only in the control medium (Figs. 3, 5). In Saccharomyces cerevisiae cells, L-proline and hydroxy-L-proline have been shown to have a protective function during endoplasmic reticulum (ER) stress in the presence of low level of amino acids, induced by nutrient deficiency. ER stress results in incorrect folding of produced proteins as well as reduction of total protein production in the cell. $S$. cerevisiae mutants without the ability to synthesise proline, are characterised by increased sensitivity to ER stress. Moreover, higher concentrations of compounds such as glutathione and NADPH were also recorded in these cells $^{44}$. It is interesting to note that oxidised and reduced glutathione were detected only in the control medium, both in case of T. rubrum and M. canis (Figs. 3, 5). Glutathione play an important role in the protection against xenobiotics, heavy metals and reactive oxygen species among aerobic prokaryotes and eukaryotes ${ }^{45}$. Moreover, in the presence of limited nutrients in the environment, glutathione is an important reservoir of nitrogen and sulphur ${ }^{45}$. Previously reports indicated also that compounds such as pyridoxine (vitamin B6) ${ }^{46}$, the metabolite marked together with glutathione in $M$. canis only in the control medium as well as para-Aminobenzoic acid $(\mathrm{PABA})^{47,48}$ and $\gamma$-aminobutyric (GABA) ${ }^{49}$ the metabolites marked in T. rubrum in the control medium may help the cell survive under stress environment such as limited nutrient availability (Fig. 3).

\section{Conclusion}

Keratin is a compact protein, and its utilisation by dermatophytes seems to be a major virulence attribute of this group of medically important fungi. The results of this study suggest that degradation of keratin produces changes in the intracellular metabolic activity of T. rubrum and M. canis. Despite some common features in the species analysed, several characteristic molecules such as cysteine, L-alanine and kynurenic acid for T. rubrum and cysteine, and riboflavin for $M$. canis were detected during keratin degradation. All these compounds appear to be important in the pathogenesis process of the fungi. The effects of this metabolomic analysis of T. rubrum and $M$. canis during keratin degradation what mimic the dermatophyte infection to the host cells, provides an opportunity to improve the knowledge available on mechanisms associated with the pathogenicity of dermatophytes and other biological properties of this group of pathogens. The next step of our research will be the identification of proteins (proteomic analysis) and whose level increases or attenuates in response to different sources of carbon (glucose and keratin) in optimum conditions of growth, which promote the adhesion of dermatophytes to host cells.

\section{Materials and methods}

Chemicals and reagents. All chemicals and reagents were LC-MS grade. Keratin originated from sheep's wool (98\% purity) was purchased from PROTEINA (Łódź, Poland). Water, acetonitrile, formic acid, and ammonium acetate were purchased from Avantor (Gliwice, Poland) and Merck (Munich, Germany). Ninety metabolites were purchased from Merck (Munich, Germany) (Table S1).

Culture conditions and extraction of intracellular metabolites. The strains T. rubrum CBS 120358 and $M$. canis CBS 11348 from Westerdijk Fungal Biodiversity Institute (formerly CBS-KNAW Collections), the Netherlands, were used in this study. Conidia from T. rubrum and M. canis strains (approximately $10^{7}$ cells $/ \mathrm{ml}$ ) were isolated as described previously by Dobrowolska and Stączek ${ }^{50}$ and germinated into YG medium contained $0.5 \%$ yeast extract as well as $2 \%$ glucose and cultivated for $72 \mathrm{~h}$ at $28^{\circ} \mathrm{C}$ with agitation ${ }^{51,52}$. Next, the cultures were transferred and incubated separately for $48 \mathrm{~h}$ at $28^{\circ} \mathrm{C}$ with agitation in liquid minimal medium (MM-Cove) at initial pH $5.0^{42}$ containing $70 \mathrm{mM}$ sodium nitrate and either $50 \mathrm{mM}$ glucose (control medium) or $0.5 \%$ (w/v) keratin as a carbon sources. Mycelia from three independent biological replicates of T. rubrum or M. canis cul- 
tures (with glucose or keratin) were collected at $48 \mathrm{~h}$ and used for metabolomic analysis. Liquid T. rubrum and $M$. canis cultures were filtered through a disposable filter with a $0.2 \mu \mathrm{m}$ pore diameter and washed using $100 \mathrm{ml}$ of deionised water. Next, $100 \mathrm{mg}$ of the wet biomass was transferred to $2 \mathrm{ml}$ LoBind tubes and frozen with liquid nitrogen. Metabolites were extracted by mechanical lysis of the entire extractant solution (biomass in $80 \%$ acetonitrile LC-MS/20\% demineralised water V/V) with frozen $3 \mathrm{~mm}$ tungsten carbide beads in a TissueLyser II homogeniser (Qiagen, Hilden, Germany) for five cycles of $1 \mathrm{~min}$ each at $30 \mathrm{~Hz}$ speed. The lysates were clarified by centrifugation at $12,000 \mathrm{rpm}$ for $5 \mathrm{~min}$ at $4^{\circ} \mathrm{C}$.

Keratinolytic assay. The keratinolytic potential of T. rubrum and M. canis was performed according to previously described protocols ${ }^{53-55}$ with modifications. In brief, $1 \times 10^{7}$ cells $/ \mathrm{ml}$ of T. rubrum and M. canis were germinated into YG medium ( $2 \% \mathrm{w} / \mathrm{v}$ glucose, $0.5 \% \mathrm{w} / \mathrm{v}$ yeast extract) for $72 \mathrm{~h}$. After the incubation time, the cultures were filtered using Falcon $40 \mu \mathrm{m}$ Cell Strainer (Corning, New York, USA) and then transferred to the $25 \mathrm{ml}$ of keratin medium (2.5 g/l) (PROTEINA, Łódź, Poland) at pH 5.0, the material was incubated for 24, 48, 72 and $96 \mathrm{~h}$ at $30^{\circ} \mathrm{C}$ under constant agitation $(120 \mathrm{rpm})$. The $\mathrm{pH}$ of medium was measured with Five Easy Plus $\mathrm{pH} / \mathrm{mv}$ bench meter (Mettler Toledo, Columbus, USA) for each day by retrieving $2 \mathrm{ml}$ liquid. Next, mycelia of $T$. rubrum and $M$. canis after 24, 48, 72 and $96 \mathrm{~h}$ of incubation were filtered using Falcon $40 \mu \mathrm{m}$ Cell Strainer (Corning, New York, USA) and the $1.0 \mathrm{ml}$ of supernatants as the enzymes were evaluated for keratynolytic activity. The reaction mixture contained $50 \mathrm{mM}$ Tris- $\mathrm{HCl}$ buffer ( $\mathrm{pH} \mathrm{8.0),} 20 \mathrm{mg}$ of keratin powder (PROTEINA, Łódź, Poland) and $1 \mathrm{ml}$ of fungal supernatant containing extracellular keratinase in total volume of $4 \mathrm{ml}$ and was incubated for $30 \mathrm{~min}$ at $45^{\circ} \mathrm{C}$ with shaking $(160 \mathrm{rpm})$. The enzyme reaction was terminating by addition of $1 \mathrm{ml}$ of $10 \%(\mathrm{w} / \mathrm{v})$ trichloroacetic acid (TCA) and cooled at $4{ }^{\circ} \mathrm{C}$ for $30 \mathrm{~min}$. After centrifugation $(10,000 \mathrm{rpm}, 15 \mathrm{~min})$, the absorbance of the supernatants was measured by spectrophotometer (Spectra Max i3, Molecular Devices, San Jose, USA) at wavelength of $595 \mathrm{~nm}$. A change of 0.01 A595 per hour equals $1 \mathrm{U}$ (unit) of keratinase activity.

LC-MS/MS conditions. Quantitative analysis of the lysates was performed using the Eksigent microLC 200 (Sciex, Framingham, Massachusetts, USA) and Agilent 1200 (Agilent, Santa Clara, California, USA) liquid chromatograph (LC) systems coupled with tandem mass spectrometer QTRAP 4500 (Sciex, Framingham, Massachusetts, USA). LC separation was carried out on three different columns in the reverse-phase mode. Vitamins, amino acids, and nucleobases were separated using a Column 1: Eksigent 3C8-EP-120 $(0.5 \times 150 \mathrm{~mm}$, $3 \mu \mathrm{m}$ ) column (Eksigent Technologies Inc., Dublin, California, USA) in positive ionisation mode (Tables S2, S3). The mobile phases consisted of $0.1 \%$ formic acid in water (mobile phase A) and $0.1 \%$ formic acid in acetonitrile (mobile phase B). Selected organic acids, pantothenate, and both forms of glutathione (reduced and oxidised) were separated using a Column 2: Eksigent C318-AQ-120 $(0.5 \times 150 \mathrm{~mm}, 3 \mu \mathrm{m})$ column Eksigent Technologies Inc., Dublin, California, USA) in negative ionisation mode (Table S2). $0.1 \%$ formic acid in water (mobile phase A) and $0.1 \%$ formic acid in acetonitrile (mobile phase B) were used as the mobile phases (Tables S2, S3). Other organic acids, nucleotides, and molecules with phosphate groups were separated using a Column 3: Synergi Hydro-RP $(2 \times 150 \mathrm{~mm}, 4 \mu \mathrm{m})$ (Phenomenex, Torrance, California, USA) in negative ionisation mode (Tables S2, S3). The mobile phases consisted of $4 \mathrm{mM}$ ammonium acetate in 95:5 acetonitrile-water (mobile phase A) and $4 \mathrm{mM}$ ammonium acetate in 5:95 acetonitrile-water (mobile phase B). The detailed conditions of the LC-MS/MS analysis of this study are available in Tables S2 and S3.

Quantitative polymerase chain reaction ( $q P C R$ ) validation. The expression patterns of eight randomly selected genes encoding the identified metabolites were analysed with primer pairs (Table 1) designed with the Primer 5 program (Premier Biosoft International, California, USA). cDNA was synthesised using $2 \mu \mathrm{g}$ of total RNA (DNA-free), RevertAid reverse transcriptase (Thermo Scientific, Waltham, MA, USA) and Random Hexamer Primers (5'-NNNNNN-3'; N = G, A, T or C) (Thermo Scientific, Waltham, MA, USA) following the manufacturer's protocol. The qPCR assay was conducted on a Rotor-Gene Q System (Qiagen, Hilden Germany) based on a method described previously ${ }^{51,52}$ using SsoAdvanced Universal SYBR Green Supermix $(2 \times)$ (Bio-Rad, Hercules, California, USA). The mixtures were subjected to an initial step at $95^{\circ} \mathrm{C}$ for $1 \mathrm{~min}$, followed by 40 cycles of denaturation at $95^{\circ} \mathrm{C}$ for $20 \mathrm{~s}$, annealing at $60.5^{\circ} \mathrm{C}$ for $20 \mathrm{~s}$, and elongation at $72{ }^{\circ} \mathrm{C}$ for $15 \mathrm{~s}$. Melting curve analysis was performed by heating the amplicon from $72{ }^{\circ} \mathrm{C}$ to $95^{\circ} \mathrm{C}$. Relative gene expression levels were calculated according to the 2- $\Delta \Delta$ CT method, with ef1- $\alpha$ and $r p l 2$ for T. rubrum, and adp-rf and $m b p-1$ for M. canis (Table 1) as the reference genes according to the MIQE (Minimum Information for Publication of Quantitative Real-Time PCR Experiments) guidelines ${ }^{56}$.

Statistical analysis. All experiments were carried out in biological and technical triplicate. Pearson's rank correlation analysis was conducted to calculate the correlation between metabolites or between species and metabolites. Differences were considered significant when $p<0.05$. After the data matrix was mean-centred and scaled to the Pareto variance, principal component analysis (PCA) was conducted with MarkerView software (Sciex, Framingham, Massachusetts, USA). The quantitative data on intracellular metabolites of T. rubrum and M. canis obtained by separation through LC-MS/MS were normalised using the Z-score algorithm on Microsoft Excel (Microsoft Corporation, Seattle, WA, USA) and heat maps were created using the GraphPad Prism 6 software (GraphPad software Inc., San Diego, California, USA). 
Received: 19 October 2020; Accepted: 4 February 2021

Published online: 17 February 2021

\section{References}

1. Zheng, H. et al. Assembly and analysis of the whole genome of Arthroderma uncinatum strain T10, compared with Microsporum canis and Trichophyton rubrum. Mycoses 63, 683 (2020).

2. Mendes, N. S. et al. Transcription of N-and O-linked mannosyltransferase genes is modulated by the pacC gene in the human dermatophyte Trichophyton rubrum. FEBS Open Bio 2, 294-297 (2012).

3. Grumbt, M. et al. Keratin degradation by dermatophytes relies on cysteine dioxygenase and a sulfite efflux pump. J. Invest. Dermatol. 133, 1550-1555 (2013).

4. Kasperova, A., Kunert, J. \& Raska, M. The possible role of dermatophyte cysteine dioxygenase in keratin degradation. Med. Mycol. 51, 449-454 (2013).

5. Tang, J. Microbial metabolomics. Curr. Genom. https://doi.org/10.2174/138920211797248619 (2011).

6. Mashego, M. R. et al. Microbial metabolomics: past, present and future methodologies. Biotech. Lett. https://doi.org/10.1007/s1052 9-006-9218-0 (2007).

7. Zhong, F., Xu, M., Metz, P., Ghosh-Dastidar, P. \& Zhu, J. A quantitative metabolomics study of bacterial metabolites in different domains. Anal. Chim. Acta 1037, 237 (2018).

8. Brandt, P., Garbe, E. \& Vylkova, S. Catch the wave: metabolomic analyses in human pathogenic fungi. PLoS Pathog. 16, e1008757 (2020).

9. Chen, F., Ma, R. \& Chen, X. L. Advances of metabolomics in fungal pathogen-plant interactions. Metabolites 9, 169 (2019).

10. Beale, D. J. et al. Untargeted metabolomics analysis of the upper respiratory tract of ferrets following influenza A virus infection and oseltamivir treatment. Metabolomics 15, 1 (2019).

11. Manchester, M. \& Anand, A. Metabolomics: strategies to define the role of metabolism in virus infection and pathogenesis. Adv. Virus Res. 98, 57 (2017).

12. Manzoni, C. et al. Genome, transcriptome and proteome: the rise of omics data and their integration in biomedical sciences. Brief. Bioinform. 19, 286 (2018).

13. Koo, I., Wei, X. \& Zhang, X. Analysis of metabolomic profiling data acquired on GC-MS. Methods Enzymol. 543, 315 (2014).

14. Vogel, C. \& Marcotte, E. M. Insights into the regulation of protein abundance from proteomic and transcriptomic analyses. Nat. Rev. Genet. 13, 227 (2012).

15. Li, X. et al. LC-MS-based metabolomic approach revealed the significantly different metabolic profiles of five commercial truffle species. Front. Microbiol. 10, 2227 (2019).

16. Soboń, A., Szewczyk, R., Różalska, S. \& Długoński, J. Metabolomics of the recovery of the filamentous fungus Cunninghamella echinulata exposed to tributyltin. Int. Biodeterior. Biodegrad. 127, 130-138 (2018).

17. Kosmides, A. K., Kamisoglu, K., Calvano, S. E., Corbett, S. A. \& Androulakis, I. P. Metabolomic fingerprinting: challenges and opportunities. Crit. Rev. Biomed. Eng. 41, 205 (2013).

18. Martinez-Rossi, N. M., Peres, N. T. A. \& Rossi, A. Pathogenesis of dermatophytosis: sensing the host tissue. Mycopathologia 182, 215 (2017).

19. Martinez, D. A. et al. Comparative genome analysis of Trichophyton rubrum and related dermatophytes reveals candidate genes involved in infection. MBio 3, 00259-12 (2012).

20. Tainwala, R. \& Sharma, Y. K. Pathogenesis of dermatophytoses. Indian J. Dermatol. 56, 259-261 (2011).

21. Mercer, D. K. \& Stewart, C. S. Keratin hydrolysis by dermatophytes. Med. Mycol. 57, 13 (2019).

22. Martins, M. P., Rossi, A., Sanches, P. R., Bortolossi, J. C. \& Martinez-Rossi, N. M. Comprehensive analysis of the dermatophyte Trichophyton rubrum transcriptional profile reveals dynamic metabolic modulation. Biochem. J. 477, 873 (2020).

23. Blechert, O., Zheng, H., Zang, X., Wang, Q. \& Liu, W. Influence of the cultivation medium and $\mathrm{pH}$ on the pigmentation of Trichophyton rubrum. PLoS ONE 14, e0222333 (2019).

24. Vylkova, S. Environmental pH modulation by pathogenic fungi as a strategy to conquer the host. PLoS Pathog. 13, e1006149 (2017).

25. Kadhim, S. K., Al-Janabi, J. K. \& Al-Hamadani, A. H. In vitro, determination of optimal conditions of growth and proteolytic activity of clinical isolates of Trichophyton rubrum. J. Contemp. Med. Sci. 1, 9-19 (2015).

26. Kadhim, S. K. \& Al-hamadani, A. H. In vitro, determination of optimal conditions of growth and proteolytic activity of clinical isolates of Trichophyton rubrum. J. Contemp. Med. Sci. 1, 9 (2015).

27. Staroń, P., Banach, M. \& Kowalski, Z. Keratin-Origins, properties, application. Chemik 65, 1019-1026 (2011).

28. Burmester, A. et al. Comparative and functional genomics provide insights into the pathogenicity of dermatophytic fungi. Genome Biol. 12, 1 (2011).

29. Bitencourt, T. A. et al. Transcription profile of Trichophyton rubrum conidia grown on keratin reveals the induction of an adhesinlike protein gene with a tandem repeat pattern. BMC Genom. 17, 1 (2016).

30. Wirthgen, E., Hoeflich, A., Rebl, A. \& Günther, J. Kynurenic Acid: The Janus-faced role of an immunomodulatory tryptophan metabolite and its link to pathological conditions. Frontiers in Immunology 8, 1957 (2018).

31. Bär, E., Whitney, P. G., Moor, K., ReiseSousa, C. \& LeibundGut-Landmann, S. IL-17 regulates systemic fungal immunity by controlling the functional competence of NK Cells. Immunity 40, 117-127 (2014).

32. Sparber, F. \& Leibundgut-Landmann, S. Interleukin-17 in antifungal immunity. Pathogens 8, 54 (2019).

33. Rouzaud, C. et al. Severe dermatophytosis and acquired or innate immunodeficiency: a review. J. Fungi 2, 4 (2016).

34. Kantún-Moreno, N. et al. Genome-Wide in silico identification of gpi proteins in mycosphaerella fijiensis and transcriptional analysis of two GPI-anchored B-1,3-glucanosyltransferases. Mycologia 105, 285-296 (2013).

35. Muszewska, A., Piłsyk, S., Perlińska-Lenart, U. \& Kruszewska, J. S. Diversity of cell wall related proteins in human pathogenic fungi. J. Fungi 4, 6 (2018).

36. Kunert, J. Physiology of keratinophilic fungi. Rev. Iberoam. Micol. 17, 77-85 (2000).

37. Dietl, A. M., Meir, Z., Shadkchan, Y., Osherov, N. \& Haas, H. Riboflavin and pantothenic acid biosynthesis are crucial for iron homeostasis and virulence in the pathogenic mold Aspergillus fumigatus. Virulence 9, 1036-1049 (2018).

38. Stahmann, K. P. et al. Riboflavin, overproduced during sporulation of Ashbya gossypii, protects its hyaline spores against ultraviolet light. Environ. Microbiol. 3, 545-550 (2001).

39. Renshaw, J. C. et al. Fungal siderophores: structures, functions and applications. Mycol. Res. 106, 1123-1142 (2002).

40. Maranhão, F. C. A., Paião, F. G. \& Martinez-Rossi, N. M. Isolation of transcripts over-expressed in human pathogen Trichophyton rubrum during growth in keratin. Microb. Pathog. 43, 166 (2007).

41. Konieczna, A., Łyżeń, R. \& Węgrzyn, G. Powiązanie glikolizy z regulacją replikacji DNA w komórkach eukariotycznych. Postepy Biochem. 61, 444-460 (2015).

42. Cove, D. J. The induction and repression of nitrate reductase in the fungus Aspergillus nidulans. Biochim. Biophys. Acta Enzymol. Biol. Oxid. 113, 51-56 (1966).

43. Rangel, D. E. N. et al. Fungal stress biology: a preface to the Fungal Stress Responses special edition. Curr. Genet. 61, 231 (2015).

44. Liang, X., Dickman, M. B. \& Becker, D. F. Proline biosynthesis is required for endoplasmic reticulum stress tolerance in Saccharomyces cerevisiae. J. Biol. Chem. 289, 27794-27806 (2014). 
45. Bakti, F. et al. Study on the glutathione metabolism of the filamentous fungus Aspergillus nidulans. Acta Microbiol. Immunol. Hung. 64, 255 (2017).

46. Bilski, P., Li, M. Y., Ehrenshaft, M., Daub, M. E. \& Chignell, C. F. Vitamin B6 (pyridoxine) and its derivatives are efficient singlet oxygen quenchers and potential fungal antioxidants. Photochem. Photobiol. 71, 129 (2007).

47. Lu, Z. et al. Para-aminobenzoic acid (PABA) synthase enhances thermotolerance of mushroom agaricus bisporus. PLoS ONE 9, e91298 (2014)

48. Wang, G. Z. et al. Proteome and transcriptome reveal involvement of heat shock proteins and indoleacetic acid metabolism process in lentinula edodes thermotolerance. Cell. Physiol. Biochem. 50, 1617 (2018).

49. Mead, O., Thynne, E., Winterberg, B. \& Solomon, P. S. Characterising the role of GABA and its metabolism in the wheat pathogen Stagonospora nodorum. PLoS ONE 8, e78368 (2013).

50. Dobrowolska, A. \& Staczek, P. Development of transformation system for Trichophyton rubrum by electroporation of germinated conidia. Curr. Genet. 55, 537-542 (2009).

51. Ciesielska, A. \& Stączek, P. Selection and validation of reference genes for qRT-PCR analysis of gene expression in Microsporum canis growing under different adhesion-inducing conditions. Sci. Rep. 8, 1197 (2018).

52. Ciesielska, A., Oleksak, B. \& Stączek, P. Reference genes for accurate evaluation of expression levels in Trichophyton interdigitale grown under different carbon sources, pH levels and phosphate levels. Sci. Rep. 9, 1 (2019).

53. Neves-da-Rocha, J. et al. Alternative splicing in heat shock protein transcripts as a mechanism of cell adaptation in Trichophyton rubrum. Cells 8, 1206 (2019).

54. Gradišar, H., Friedrich, J., Križaj, I. \& Jerala, R. Similarities and specificities of fungal keratinolytic proteases: comparison of keratinases of Paecilomyces marquandii and Doratomyces microsporus to some known proteases. Appl. Environ. Microbiol. 71, 3420-3426 (2005).

55. Zhang, X. et al. Metalloprotease genes of Trichophyton mentagrophytes are important for pathogenicity. Med. Mycol. 52, 36 (2014).

56. Bustin, S. A. et al. The MIQE guidelines: minimum Information for publication of quantitative real-time PCR experiments. Clin. Chem. 55, 611-622 (2009).

\title{
Acknowledgements
}

This study was supported by Grant No. 2016/23/D/NZ7/03964 from the National Science Centre, Poland.

\section{Author contributions}

A.C. conceived and conducted the experiments, analysed the data, interpreted the results, and wrote the manuscript. A.K. conducted the experiments. K.K. conducted the experiments. A.S. conceived the experiments. R.S. conceived the experiments. All authors reviewed the manuscript.

\section{Funding}

This work was supported by Grant 2016/23/D/NZ7/03964 from National Science Centre, Poland.

\section{Competing interests}

The authors declare no competing interests.

\section{Additional information}

Supplementary Information The online version contains supplementary material available at https://doi. org/10.1038/s41598-021-83632-z.

Correspondence and requests for materials should be addressed to A.C.

Reprints and permissions information is available at www.nature.com/reprints.

Publisher's note Springer Nature remains neutral with regard to jurisdictional claims in published maps and institutional affiliations.

\begin{abstract}
Open Access This article is licensed under a Creative Commons Attribution 4.0 International License, which permits use, sharing, adaptation, distribution and reproduction in any medium or format, as long as you give appropriate credit to the original author(s) and the source, provide a link to the Creative Commons licence, and indicate if changes were made. The images or other third party material in this article are included in the article's Creative Commons licence, unless indicated otherwise in a credit line to the material. If material is not included in the article's Creative Commons licence and your intended use is not permitted by statutory regulation or exceeds the permitted use, you will need to obtain permission directly from the copyright holder. To view a copy of this licence, visit http://creativecommons.org/licenses/by/4.0/.
\end{abstract}

(C) The Author(s) 2021 